\title{
Maternal serum adipokines and inflammatory markers at late gestation and newborn weight in mothers with and without gestational diabetes mellitus
}

\author{
Renata Saucedo ${ }^{1} \mathbb{D}$, Jorge Valencia ${ }^{1} \mathbb{D}^{\circ}$, Luz Elena Moreno-González ${ }^{1} \mathbb{D}$, \\ María Isabel Peña-Cano ${ }^{2}{ }^{\infty}$, Alejandra Aranda-Martínez ${ }^{1} \mathbb{D}$, Yolanda García ${ }^{1} \mathbb{D}$, \\ Mary Flor Díaz-Velázquez ${ }^{3}$ (1) , Marcelino Hernández-Valencia ${ }^{1}$ (1) \\ ${ }^{1}$ Endocrine Research Unit, National Medical Center, Instituto Mexicano del Seguro Social, Mexico City, Mexico \\ ${ }^{2}$ Faculty of Chemistry, Universidad Autonoma del Estado de Mexico, State of Mexico, Mexico \\ ${ }^{3}$ Hospital of Gynecology and Obstetrics 3, Medical Center La Raza, Instituto Mexicano del Seguro Social, Mexico City, Mexico
}

\begin{abstract}
Objectives: Maternal obesity increases the risk of gestational diabetes mellitus (GDM) and is positively correlated with neonatal obesity increasing the risk of adiposity in both young and adult offspring. Maternal secreted factors from adipose tissue such as adipokines and inflammatory cytokines may regulate fetal growth. This study investigated associations between maternal adipokines and inflammatory markers at late gestation, and neonatal anthropometric characteristics in mothers with and without GDM.

Material and methods: The study included 65 women with GDM and 65 pregnant women with normal glucose tolerance evaluated at the time of term elective Caesarean section. Adiponectin, leptin, resistin, adipsin, neutrophil gelatinase-associated lipocalin (NGAL), nerve growth factor (NGF), monocyte chemotactic protein-1 (MCP-1) and tumor necrosis factor-alpha (TNF-alpha) concentrations were measured in maternal serum by the multiplex immunoassay using Magpix technology. C-reactive protein (CRP) was measured with a particle-enhanced turbidimetric immunoassay and neonatal anthropometric variables were assessed. The association of birthweight with individual biomarkers was analyzed using multivariate logistic regression adjusted for maternal factors.

Results: Adiponectin, leptin, resistin, adipsin, NGAL and NGF were not significantly associated with higher birthweight. The maternal factors in association with higher birthweight observed in GDM were CRP, MCP-1 and TNF-alpha levels. Regression analysis showed that TNF-alpha was an independent risk factor for higher birthweight $(p=0.046)$.

Conclusions: These results suggest an involvement of maternal inflammatory markers at late gestation and fetal growth in mothers with GDM, and that TNF-alpha could play a major role.
\end{abstract}

Key words: gestational diabetes mellitus; adipokines; cytokines; maternal obesity; birthweight

Ginekologia Polska 2022; 93, 2: 126-133

\section{INTRODUCTION}

Obesity is a major clinical problem in women of reproductive age worldwide [1]. It is currently estimated that three in four women of reproductive age in Mexico are classified as overweight or obese [2]. Maternal obesity increases the risk of infertility, stillbirth, gestational diabetes mellitus (GDM), preeclampsia, pregnancy-induced hypertension, thromboembolism and complications during delivery. In addition, maternal obesity is positively correlated with neonatal and childhood obesity in the offspring with negative consequences that include increased risk of metabolic syndrome and type 2 diabetes mellitus (T2DM) later in life [3].

The prevalence of GDM, which is a state of glucose intolerance that develops during pregnancy, is rising worldwide [4]. In Mexico, it ranges from $10.3 \%$ to $30.1 \%$, depending on the diagnostic criteria used [5, 6]. GDM increases the risk of T2DM, and associate with higher risk of large for gestational age (LGA) products, who subsequently develop an increased 
risk of obesity and diabetes in adulthood [7]. It is established that the transport of nutrients other than glucose from the mother to the fetus as amino acids and/or lipids could contribute to excessive fetal growth [8].

As pregnancy increases adiposity, attention has been drawn to the role of maternal factors associated with adipose tissue (adipokines and inflammatory markers) in mediating fetal growth [9-12]. Adiponectin, the most commonly found adipokine, is produced exclusively by adipose tissue and is suggested to have various properties, including insulin-sensitizing, anti-atherogenic, and anti-inflammatory. Deficient concentrations in plasma of adiponectin correlate with GDM and obesity [13]. This may be due to reduced levels of adiponectin causing a reduction of glucose uptake in skeletal muscle, accompanied by an increase in production of hepatic glucose. In addition, studies have shown that maternal adiponectin is inversely associated with birthweight $[14,15]$.

Leptin is released into the circulation in white adipose tissue in proportion to the amount of lipid stores and it has been suggested that increased maternal leptin concentrations in the third trimester of pregnancy may be attributed to the placenta, and not maternal adipose tissue. Leptin regulates placental growth, nutrient transfer, angiogenesis, and trophoblast invasion and enhances the mobilization of maternal fat stores to the fetus. Moreover, most studies have found increased leptin concentrations in GDM [13] and maternal leptin levels have been shown to be associated to fetal growth, and an association between maternal leptin levels during pregnancy and offspring adiposity at two years of age has also been observed [16].

Tumor necrosis factor-alpha (TNF-alpha) is a multifunctional cytokine, and a central regulator of inflammation. TNF-alpha is defined as a cytokine which is the product of monocytes, macrophages, T-cells, neutrophils, fibroblasts and adipocytes. TNF-alpha induces insulin resistance in skeletal muscle and adipose tissue. Plasma TNF-alpha levels correlate with body mass index (BMI) and insulin resistance. High TNF-alpha levels from early gestation are associated with subsequent GDM [13]. There is also evidence that TNF-alpha transcript is significantly increased in placentas from women with GDM at term, compared to non-diabetic control women [17]. It appears that TNF-alpha can activate docosahexaenoic acid, an essential $\omega-3$ polyunsaturated fatty acid that accumulates in placenta of offspring from mothers with GDM, causing increased adiposity in newborns [18].

C-reactive protein (CRP) is a nonspecific marker of inflammation in the body that increases gradually throughout pregnancy to approaching labor. Monocyte chemotactic protein-1 (MCP-1) is a chemokine secreted by monocytes, macrophages, lymphocytes, endothelial cells, decidua, myo- metrium and placenta. It is a chemotactic factor that attracts and activates monocytes and macrophages into sites of inflammation. MCP-1 increases during normal pregnancy and even more during labor, suggesting that MCP-1 modulates the immune system as pregnancy advances. Several studies have reported that maternal serum CRP and MCP-1 concentrations are negatively correlated to birthweight $[19,20]$.

Adipsin is a protease with close homology to human complement $\mathrm{D}$. It is secreted from muscle, lung, peripheral nerves, placenta and adipose tissue. Adipsin is the rate-limiting enzyme in the formation of acylation stimulating protein, a factor contributing to lipid storage in adipose tissue. Adipsin levels are significantly higher in obesity and positively related to BMI. Furthermore, higher levels of adipsin have been reported in GDM [21]. A recent study found no association between cord blood adipsin and birthweight [22].

Neutrophil gelatinase-associated lipocalin (NGAL), also known as lipocalin 2, is a glycoprotein secreted by neutrophils, epithelial cells and adipocytes. It is abundantly present in damaged epithelia during inflammation and cardiovascular disease. NGAL concentrations are elevated in obesity, T2DM and GDM [23].

Resistin is a pro-inflammatory adipokine expressed in adipocytes, pancreatic islets, mononuclear cells, macrophages, placenta and liver. In rodents, resistin represents a clear pathogenic factor in the severity of insulin resistance. However, in humans, this adipokine correlates with insulin resistance as a consequence of obesity. Some studies have suggested that elevated circulating resistin is a risk factor for GDM. One study found mRNA expression of gene-encoded resistin was increased in adipose tissue from GDM when compared to a non-GDM group, and the expression level was related to insulin resistance [24]. Conflicting findings have been found regarding cord serum resistin and birthweight $[25,26]$.

Nerve growth factor (NGF) is one of the neurotrophic factors, and associates with the survival, development and function of central nervous system basal forebrain cholinergic neurons, as well as peripheral embryonic and sympathetic sensory neurons. In addition, NGF appears to play an important role in placental and fetal growth. In small for gestational age (SGA) infants born at term, NGF levels are markedly higher than appropriate for gestational age (AGA) and LGA infants [27]. The NGF gene is also found in white adipose tissues, and it increases in the presence of inflammatory cytokines, including TNF-alpha. In addition, levels of circulating NGF are upregulated in obesity and metabolic syndrome [28].

In summary, most studies related to maternal adipokines and birthweight have yielded inconsistent findings. In addition, very few studies have investigated maternal CRP, 
MCP-1, resistin, adipsin, NGAL and nerve growth factor NGF levels. The aim of this study was to determine whether there is an association between maternal plasma adiponectin, leptin, resistin, adipsin, NGAL, NGF, CRP, MCP-1 and TNF-alpha concentrations at late gestation, GDM, and the fetal growth.

\section{MATERIAL AND METHODS \\ Research Design and Study Population}

Data for this cross-sectional study was obtained from a larger study of women who were recruited from the Hospital of Gynecology and Obstetrics 3, Medical Center La Raza, IMSS (Mexico City), or the Hospital of Gynecology and Obstetrics 221, IMSS (Toluca, State of Mexico), for an elective caesarean section, for a study on adipokine gene expression in omental adipose tissue in GDM (data not published). The original study was approved by the Institutional Review Board of the Instituto Mexicano del Seguro Social (IMSS) in Mexico City (R-2018-785-026) and all participants gave written informed consent. The study included singleton births to mothers over 18 years of age from January 2019 to December 2019. To eliminate any confounding effects from the labor process, only those undergoing a term (37-41 weeks of gestation) elective Caesarean section were considered for the study (indications for Caesarean included breech presentation, previous Caesarean section, and/or when macrosomia was suspected by ultrasonography at 38 weeks of gestation). Women with pre-gestational diabetes or hypertension and autoimmune, immunosuppressive, kidney, heart, infectious diseases or smoking and alcohol habits as well as pregnancies complicated by fetal anomalies were excluded from the study. The present study included term births to both normal glucose tolerant (NGT) women and women with GDM. Gestational age was estimated by last menstrual period and confirmed by ultrasonographic measurements at first trimester in all subjects. All pregnant women were screened for GDM at 24-28 weeks of gestation and were classified according to the results of the screening. Women with GDM were diagnosed according to the International Association of Diabetes and Pregnancy Study Groups criteria by one or more abnormal glucose value during a $75 \mathrm{~g}$-oral glucose tolerance test (OGTT), with fasting levels $\geq 5.1 \mathrm{mmol} / \mathrm{L}$, $1 \mathrm{~h} \geq 10 \mathrm{mmol} / \mathrm{L}$ or $2 \mathrm{~h} \geq 8.5 \mathrm{mmol} / \mathrm{L}$. Management after GDM diagnosis was started with medical nutrition therapy (1600-1800 kcal/day with restriction of carbohydrates to $35-40 \%$ ), and moderate physical activity (30 minutes of moderate-intensity aerobic exercise at least five days a week) and subsequent evaluation of glycemic control with fasting glucose and postprandial blood glucose at two hours after meals at 2-4 week intervals. Insulin therapy (0.7-1.0 units/kg of body weight daily) was prescribed for women who did not achieve glycemic control with diet (fasting glucose levels $<5.27 \mathrm{mmol} / \mathrm{L}$, and postprandial blood glucose values $<6.66 \mathrm{mmol} / \mathrm{L}$ at 2 hours). Of the women with GDM, 75\% ( $n=49)$ were treated by diet and moderate physical activity only, and the remainder $(n=16)$ received the aforementioned insulin therapy for glycemic control.

Information about current and previous pregnancies was collected on a baseline questionnaire using medical records. Demographic characteristics included maternal age, history of GDM and family history of diabetes in a first-degree relative. Maternal pre-pregnancy BMI was calculated from patient-reported pre-pregnancy weight and the height measured during the first visit of gestation. BMI at the time of delivery was also calculated.

\section{Birth size measures}

Anthropometric measures (weight, length, foot length, head circumference, chest circumference, abdominal circumference, and ponderal index) were assessed at birth in the term infants. Ponderal index was calculated as $100 \times$ [birthweight $(\mathrm{g}) /$ length $\left.\left(\mathrm{cm}^{3}\right)\right]$. The infants were classified as small for gestational age (SGA), appropriate for gestational age (AGA) or large for gestational age (LGA) according to weight for gestational age of Mexican children [29]. LGA and SGA were defined as $>90^{\text {th }}$ and $<10^{\text {th }}$ percentile, respectively. Macrosomia was considered in cases of birth weight $\geq 4000 \mathrm{~g}$. Information about the infant's sex, and Apgar scores came from birth certificates. Apgar scores were noted soon after delivery at one minute and at five minutes.

\section{Biochemical analysis}

Maternal fasting blood samples for biochemical analysis were obtained by venipuncture together with the samples for routinely performed laboratory tests at the day of the scheduled Caesarean section. The samples could clot for at least 30 minutes before centrifugation at $1000 \mathrm{~g}$, which was continued for 15 minutes. Serum aliquots were frozen at $-70^{\circ}$ C until assayed. Levels of glucose, total cholesterol, high-density lipoprotein (HDL) cholesterol and triglycerides were measured in fresh samples on an ARCHITECT Plus c4000 Clinical Chemistry Analyzer (Abbot Diagnostics, Abbott Park, IL, USA). Levels of low- density lipoprotein (LDL) cholesterol were estimated with use of the Friedewald formula. CRP was measured with a particle-enhanced turbidimetric immunoassay (MULTIGENT CRP Vario kit; Sentinel CH, Milan, Italy) on the ARCHITECT Plus c4000 Clinical Chemistry Analyzer (Abbot Diagnostics, Abbott Park, IL, USA). Adiponectin, leptin, resistin, adipsin, NGAL, NGF, MCP-1, TNF-alpha, and insulin were measured through the multiplex immunoassay using Magpix technology (Milliplex Map, Billerica, MA, USA). Insulin resistance was calculated using the homeostasis model as- 
sessment for insulin resistance (HOMA-IR) method, where HOMA-IR = fasting insulin concentration $[\mu \mathrm{U} / \mathrm{mL}] \times$ fasting glucose concentration [mmol/L]/22.5 [30].

\section{Statistical analysis}

Kolmogorov-Smirnov test was used to assess data distribution. Results are presented as medians with interquartile range (IQR). Differences between groups were analyzed by Mann-Whitney test and Kruskal-Wallis test. To assess the correlation of the data, Spearman's correlation test was performed. Logistic regression analysis with LGA as a dependent variable was performed with adjustment for the following covariates: length of gestation, infant sex, maternal demographic factors, maternal anthropometric measures, plasma glucose levels, lipids, insulin, adipokines and inflammatory proteins. We used IBM SPSS Statistics 23.0 (IBM SPSS Inc., Chicago, IL) for statistical analysis, and $\mathrm{p}<0.05$ was defined as significant.

\section{RESULTS}

The demographic and clinical data of mothers are shown in Table 1. Women with GDM had a higher age, weight,
BMI, and parity, previous GDM, family history of diabetes and higher glucose levels at OGTT screening and glucose concentration at late gestation than women with normal glucose tolerance. They did not significantly differ from women without GDM in maternal weight gain at the time of term elective Caesarean section, total cholesterol, triglycerides, $\mathrm{HDL}, \mathrm{LDL}$, insulin and HOMA-IR.

Results of the adipocitokines are presented in Table 2. There were no significant differences in adiponectin, leptin, resistin, NGAL, NGF, and CRP levels between subjects with GDM and controls. Women with GDM had higher adipsin, MCP-1, and TNF-alpha levels. These observed differences did not remain after adjusting for age and weight, and insulin treatment did not significantly affect adipokine levels between women taking insulin therapy and those not.

Elevated pre-pregnancy maternal BMI in GDM and NGT subjects, was found to be positively associated with $\operatorname{adipsin}(r=0.320, p=0.002)$, leptin $(r=0.369, p=0.001)$, MCP-1 $(r=0.410, p=0.001)$, and TNF-alpha levels $(r=0.341$, $\mathrm{p}=0.001)$.

Gestational age at delivery was significantly lower among GDM in comparison with healthy pregnant women (Tab. 3).

\begin{tabular}{|c|c|c|c|}
\hline & NGT $(n=65)$ & GDM $(n=65)$ & p \\
\hline Maternal age [years] & $26(22-31)$ & $32(28-35)$ & 0.001 \\
\hline Pregravid weight [kg] & $60.0(54.8-67.0)$ & $78.1(63.5-96.5)$ & 0.001 \\
\hline Weight at delivery [kg] & $70.3(65.7-77.3)$ & $85.2(72.5-104.5)$ & 0.001 \\
\hline Pre-pregnancy BMI [kg/m²] & $25.1(23.0-27.1)$ & $32.5(26.3-38.3)$ & 0.001 \\
\hline BMI at delivery $\left[\mathrm{kg} / \mathrm{m}^{2}\right]$ & $29.6(27.0-31.5)$ & $34.8(30.8-41.4)$ & 0.001 \\
\hline Maternal weight gain [kg] & $10.2(7.1-12.5)$ & $8.3(4.2-12.3)$ & 0.202 \\
\hline $\begin{array}{l}\text { Parity n [\%] } \\
\text { Nulliparous } \\
\text { One or greater }\end{array}$ & $\begin{array}{l}24(36.9) \\
41(63.1)\end{array}$ & $\begin{array}{c}9(13.8) \\
56(86.2)\end{array}$ & 0.008 \\
\hline Family history of diabetes n [\%] & $7(10.8)$ & $23(35.4)$ & 0.004 \\
\hline Past history of GDM n [\%] & $0(0.0)$ & $12(18.5)$ & 0.001 \\
\hline \multicolumn{4}{|l|}{ Blood glucose at OGTT [mmol/L] } \\
\hline Fasting & $4.0(3.8-4.2)$ & $5.4(4.6-5.8)$ & 0.001 \\
\hline $1 \mathrm{~h}$ & $7.5(6.5-8.8)$ & $12.8(11.0-13.0)$ & 0.001 \\
\hline $2 \mathrm{~h}$ & $6.4(5.5-7.4)$ & $9.8(8.7-10.5)$ & 0.005 \\
\hline Fasting glucose at delivery [mmol/L] & $4.2(3.8-4.8)$ & $4.7(4.1-5.3)$ & 0.05 \\
\hline Triglycerides at delivery [mmol/L] & $2.9(2.5-3.6)$ & $3.4(2.2-3.9)$ & 0.280 \\
\hline $\mathrm{HDL}$ at delivery $[\mathrm{mmol} / \mathrm{L}]$ & $2.5(2.2-3.0)$ & $2.4(1.9-3.0)$ & 0.319 \\
\hline LDL at delivery [mmol/L] & $1.8(1.3-2.5)$ & $1.4(0.84-2.1)$ & 0.081 \\
\hline Total cholesterol at delivery $[\mathrm{mmol} / \mathrm{L}]$ & $60.1(52.0-67.0)$ & $55.8(49.3-66.0)$ & 0.188 \\
\hline Fasting insulin at delivery $[\mathrm{mmol} / \mathrm{L}]$ & $56.0(37.6-82.7)$ & $60.4(43.4-81.7)$ & 0.902 \\
\hline HOMA-IR at delivery & $1.4(0.9-2.3)$ & $1.7(1.3-2.5)$ & 0.149 \\
\hline
\end{tabular}

Data are presented as medians (interquartile range) as well as counts and percentages; NGT — normal glucose tolerant; GDM — gestational diabetes mellitus; BMI — body mass index; OGTT — oral glucose tolerance test; HDL — high-density lipoprotein cholesterol; LDL — low- density lipoprotein cholesterol; HOMA-IR — homeostasis model assessment-insulin resistance 
Table 2. Maternal adipokines and inflammatory markers for normal pregnancy and GDM at delivery

\begin{tabular}{|l|c|c|c|}
\hline & NGT $(\mathbf{n}=\mathbf{6 5})$ & GDM $(\mathbf{n}=\mathbf{6 5})$ & $\mathbf{p}$ \\
\hline Adiponectin [pg/mL] & $213.5(77.6-766.4)$ & $220.9(64.6-605.1)$ & 0.490 \\
\hline Resistin $[\mathrm{pg} / \mathrm{mL}]$ & $54.6(38.6-68.8)$ & $43.9(32.4-62.9)$ & 0.097 \\
\hline Adipsin $[\mathrm{pg} / \mathrm{mL}]$ & $2.5(1.9-3.1)$ & $3.1(2.4-3.9)$ & 0.011 \\
\hline NGAL $[\mathrm{pg} / \mathrm{mL}]$ & $164.9(112.0-234.7)$ & $159.7(109.7-247.2)$ & 0.520 \\
\hline NGF $[\mathrm{pg} / \mathrm{mL}]$ & $2.1(1.6-2.6)$ & $2.1(1.6-2.9)$ & 0.487 \\
\hline Leptin $[\mathrm{pg} / \mathrm{mL}]$ & $7.4(4.7-11.3)$ & $8.4(4.9-17.0)$ & 0.538 \\
\hline CRP $[\mathrm{nmol} / \mathrm{L}]$ & $6.9(3.7-10.8)$ & $9.5(5.0-22.9)$ & 0.096 \\
\hline MCP-1 $[\mathrm{pg} / \mathrm{mL}]$ & $93.3(68-137)$ & $126.5(102.4-165.2)$ & 0.005 \\
\hline TNF-alpha $[\mathrm{pg} / \mathrm{mL}]$ & $2.9(2.3-3.5)$ & $3.9(2.8-4.7)$ & 0.006 \\
\hline
\end{tabular}

Data are presented as medians (interquartile range); NGT — normal glucose tolerant; GDM — gestational diabetes mellitus; NGAL — neutrophil gelatinase-associated lipocalin; NGF - nerve growth factor; CRP - C-reactive protein; MCP-1 - monocyte chemotactic protein-1; TNF — alpha, tumor necrosis factor-alpha

Table 3. Neonatal characteristics grouped by maternal glucose tolerance status during pregnancy

\begin{tabular}{|l|c|c|c|}
\hline & NGT (n= 65) & GDM (n= 65) & p \\
\hline Gestational age [weeks] & $39(38-40)$ & $38(38-39)$ & 0.013 \\
\hline Sex, female & $30(46.2)$ & $41(63.0)$ & 0.109 \\
\hline Birth weight [g] & $3100(2875-3300)$ & $3400(3000-3900)$ & 0.001 \\
\hline Birth length [cm] & $49(48-50)$ & $50(49-52)$ & 0.037 \\
\hline Ponderal index [g/cm $\left.{ }^{3}\right]$ & $2.5(2.4-2.76)$ & $2.7(2.5-2.95)$ & 0.05 \\
\hline Nenonatal foot length [cm] & $8(7-8)$ & $8(7-8)$ & 0.560 \\
\hline Neonatal head circumference [cm] & $34(33.25-35)$ & $35(34-36)$ & 0.084 \\
\hline Neonatal chest circumference $[\mathrm{cm}]$ & $33(32-34)$ & $32(31-34)$ & 0.510 \\
\hline Neonatal abdominal circumference $[\mathrm{cm}]$ & $30(30-32)$ & $31(29-33)$ & 0.412 \\
\hline $\begin{array}{l}\text { Birth weight for gestational age } \mathrm{n}[\%] \\
\text { Appropriate for gestational age } \\
\text { Large for gestational age }\end{array}$ & $63(97)$ & & 0.001 \\
\hline Macrosomia $n$ [\%] & $2(3)$ & $44(68)$ & 0.001 \\
\hline Apgar 1'Score & $1(0.65)$ & $21(32)$ & 0.639 \\
\hline Apgar 2' Score & $8(8-8)$ & $10(6.5)$ & 0.519 \\
\hline
\end{tabular}

Data are presented as medians (interquartile range) as well as counts and percentages; NGT — normal glucose tolerant; GDM — gestational diabetes mellitus

However, offspring of mothers with GDM had higher weight, height, and ponderal index than offspring of mothers without GDM. The rate of LGA was significantly higher in the GDM group than in patients with a non-GDM pregnancy. The occurrence of macrosomia was markedly higher in GDM group; there were ten macrosomic newborns in GDM and one in the NGT group. The rate of girls, foot length, head circumference, chest circumference, abdominal circumference, and Apgar score did not significantly differ between both groups.

Pre-pregnancy BMI, biochemical maternal parameters and adiponectin, leptin, resistin, adipsin, NGAL and NGF were not significantly associated with higher birthweight.
The maternal factors in association with higher birthweight observed were CRP, MCP-1, and TNF-alpha levels. Among women with GDM, the median maternal plasma concentrations of CRP and MCP-1 showed no significant differences between mothers of AGA and LGA neonates (Fig. 1). However, the median maternal plasma CRP and MCP-1 concentration were higher in mothers of LGA neonates with GDM than in mothers with NGT and AGA newborns. Furthermore, among women with GDM, the median maternal plasma TNF-alpha concentration was higher in women with an LGA neonate than in those with an AGA newborn, and TNF-alpha concentration was higher in women with GDM and LGA neonate than in those with NGT and AGA newborn. 

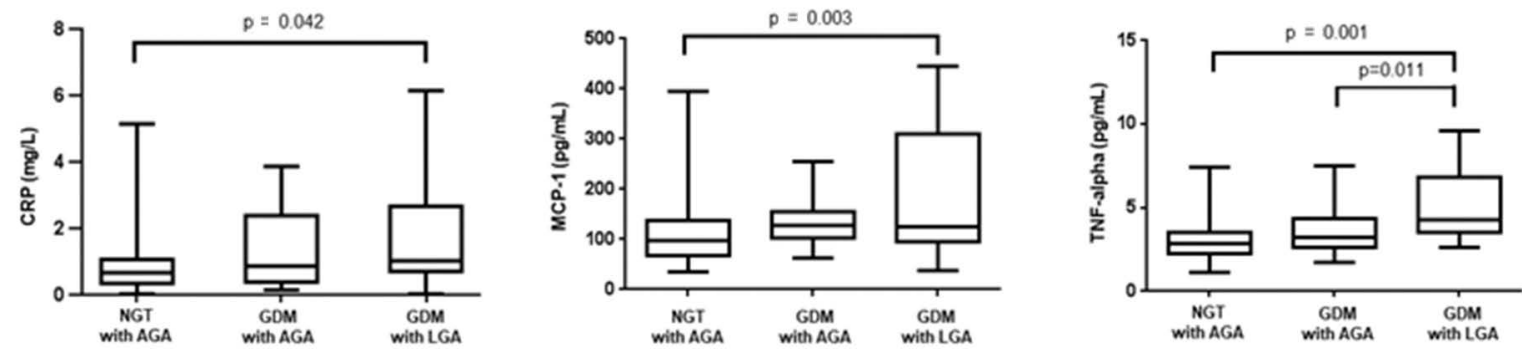

Figure 1. Box and whisker plots of serum CRP, MCP-1 and TNF-alpha levels between the study groups and/or cases of an LGA product. Among women with GDM, there were no statistically significant differences in concentrations of CRP and MCP-1 in maternal plasma between patients with AGA and LGA products. However, the median maternal plasma CRP and MCP-1 concentration were higher in pregnant women with GDM and LGA neonates than in those with NGT and AGA newborns. Furthermore, among women with GDM, the maternal plasma concentrations of TNFalpha were higher in cases of GDM and LGA neonate than in NGT and AGA newborn; NGT — normal glucose tolerant; GDM — gestational diabetes mellitus; AGA — appropriate for gestational age; LGA — large for gestational age; CRP — C-reactive protein; MCP-1 - monocyte chemotactic protein-1;TNF-alpha - tumor necrosis factor-alpha

On logistic regression analysis, the significant predictor of having a LGA infant, after adjustment for covariates, was TNF-alpha level (odds ratio: 1.55, 95\% confidence interval: 1.04-2.32, $\mathrm{p}=0.046$ ).

\section{DISCUSSION}

In our study, we evaluated the association between specific maternal adipokines and inflammatory markers at late gestation and fetal growth in mothers with GDM. Notably, offspring of mothers with GDM had higher weight, height and ponderal index than offspring of mothers without GDM, and the rate of LGA was significantly higher in the GDM group than in patients with a non-GDM pregnancy. Gestational diabetes mellitus is a common predisposing factor for having infants who are born large for gestational age and subsequently develop an increased risk of obesity and T2DM in adulthood [7]. Maternal blood glucose level is associated with birthweight. However, even with strict glycemic control, women with GDM have higher frequency of macrosomic infants [31]. It has been suggested that other nutrients, such as triglycerides and amino acids, contribute to excessive fetal growth [8]. In this study, we did not find any relation of either maternal fasting glucose or triglyceride concentration at term with newborn weight. Similarly, none of the metabolic measures, such as glucose values at OGTT, total cholesterol, HDL, LDL, insulin and HOMA-IR, were associated with birth weight. Moreover, although mean values of OGTT indices at screening were significantly elevated in GDM over healthy controls, indicating increased insulin resistance in GDM group, there was no difference between studied groups regarding lipid profile, insulin and HOMA-IR at term. This could be a consequence of metabolic control performed in GDM, which was evaluated at 2-4-week intervals until delivery. Notably, maternal weight gain during pregnancy did not differ between the study groups.
Recently, maternal adipokines and inflammatory cytokines have been identified as independent risk determinants of fetal overgrowth [9-12]. In the present study, fetal growth was associated with higher CRP, MCP-1, and TNF-alpha levels in mothers with GDM. The association between newborn weight and TNF-alpha was independent of established risk factors of GDM such as age, prepregnancy BMI, parity, and family history of T2DM. Similarly, recent findings from Kumarathasan et al., demonstrated a proinflammatory status in LGA healthy mothers, as higher inflammatory index compared to the AGA group [19].

Some studies have reported, contrary to our finding, that maternal serum CRP and MCP-1 concentrations are negatively correlated to birthweight. Thus, CRP at 28 weeks' gestation in healthy pregnant women, as well as maternal MCP-1 at third trimester (32-34 weeks), were associated with SGA births $[19,20]$. The discrepancy between our finding and others might be related to gestational age at sampling. Further, our population differs from others in relation to GDM, which could affect the maternal inflammatory state. GDM associates with changes in inflammatory profiles, both maternal, fetal and placental, mirrored by an increase in circulating inflammatory molecules [32].

Obesity is associated with pathologic sequelae, including chronic inflammation and adipocyte dysregulation. Maternal obesity, defined by an elevated pre-pregnancy BMI, is a risk factor for fetal macrosomia. In our study, maternal BMI was not associated with birthweight. However, maternal BMI was in correlation with the serum adipsin, leptin, MCP-1, and TNF-alpha levels, suggesting that one of the main sources of cytokine production is the adipose tissue, although it has also been documented that the feto-placental unit could also be an important source of TNF-alpha and leptin $[13,17]$.

In the current study, we did not find an association between maternal adipokines adiponectin, leptin, adipsin 
NGAL, NGF and resistin levels and fetal growth. Previous data have shown that some of these adipokines are associated with fetal growth. However, most studies have examined maternal adipokines in midpregnancy, or are focused on cord blood. In our study, maternal adipokines were measured at the time of Caesarean section. It has been demonstrated that blood levels of adipokines change throughout pregnancy in relation to the increased fat accretion in the first two trimesters of pregnancy and to the changes in clearance in latter stages of pregnancy. In addition, the gestational diabetes participants in our study had higher pre-pregnancy BMI, parity, and birthweight than pregnant controls, which are factors associated with higher circulating blood volume. The increase in plasma volume would result in lower circulating adipokine levels. Other discrepancies may also be due to the different genetic backgrounds or environmental condition of the populations studied, study design, sample size of the population, the diagnosis criteria of GDM patients, the status of glycemic control, and the assay methods used to measure adipokines.

The cross-sectional design together with the limited sample size, must be considered as a limitation of our study. We did not assess the associations between the adipokines at various points of time in gestation and birth outcomes. We also relied on self-reported pre-pregnancy weight, which is susceptible to reporting bias. Besides, the difference in age and BMI between women with GDM and women with a non-GDM pregnancy must be considered, although adjustments for these variables were made. In addition, there was a lack of $\mathrm{HbA} 1 \mathrm{C}$, and cord plasma measurements and of other, more specific, measures of maternal adiposity, that is, intra-abdominal maternal visceral adipose tissue (VAT). BMI conveys no information about the quantity, quality, location, or metabolic function of fat depots. Measurement of VAT by ultrasound in early pregnancy strongly predicts GDM and is associated with birthweight [33, 34]. Excess VAT leads to increased release of fatty acids and secretion of pro-inflammatory substances, with consequent changes in fetal growth.

Strengths of this study include the assessment of several maternal adipokines, while much of the prior literature is focused on cord blood or neonatal blood and the control of numerous pregnancy factors, including mode of delivery, gestational age, maternal fasting status at delivery and maternal smoking.

Overall, the major present findings reveal that circulating maternal CRP, MCP-1, and TNF-alpha are higher in term GDM and LGA neonates than in those with term NGT and AGA newborns. Although it is not possible to determine exactly if the source of these inflammatory markers is placenta or adipose tissue, this provides a further evidence for a possible association of these inflammatory markers with metabolic processes in the feto-placental unit involved in fetal growth. This inflammatory profile can alter developmental programming and have long-lasting influence on offspring. In support of this, Perrin et al. [35], in a cohort of infants born prior to 28-weeks' gestation, reported that obesity at age two years is predicted by perinatal systemic inflammation.

\section{CONCLUSIONS}

These results suggest an involvement of maternal inflammatory markers at late gestation and fetal growth in mothers with GDM, and that TNF-alpha could play a major role. Further mechanistic studies are necessary to investigate the precise roles of these inflammatory markers in birthweight.

\section{Acknowledgements}

We thank the Hospital of Gynecology and Obstetrics 3, Medical Center La Raza, Instituto Mexicano del Seguro Social (Mexico City) and the Hospital of Gynecology and Obstetrics 221, Instituto Mexicano del Seguro Social (Toluca, State of Mexico) for providing patient care services. We also thank Chemist Luis Enrique Tenorio Vieyra and Chemist Sandra Campos for performing the biochemical assays, and Dr. Erubiel Rosendo Luis López, Dr. Lizbeth Chinolla Arellano, and Dr. Edgar Mendoza Reyes for providing access to patients.

\section{Funding}

This study was supported by scientific grants from Instituto Mexicano del Seguro Social (FIS/IMSS/PROT/G18/1826). RS and MHV hold a fellowship from the National System of Investigators (Consejo Nacional de Ciencia y Tecnología).

\section{Authors' contributions}

RS, JV, and MHV planned and designed the study. JV, MIPC, LEMG, AAM, and YG performed laboratory biomarker analysis. RS, JV, and MFDV drafted the manuscript and RS performed the data analysis. All authors provided significant intellectual contribution in interpreting data and critical review of the manuscript. All authors approved the final version of the manuscript.

\section{Conflict of interests}

The authors declare that they have no competing interests.

\section{REFERENCES}

1. Poston L, Caleyachetty R, Cnattingius S, et al. Preconceptional and maternal obesity: epidemiology and health consequences. Lancet Diabetes Endocrinol. 2016; 4(12): 1025-1036, doi: 10.1016/S2213-8587(16)302170 , indexed in Pubmed: 27743975.

2. Encuesta Nacional de Salud y Nutrición (ENSANUT) 2018. Presentación de Resultados. https://ensanut.insp.mx/encuestas/ensanut2018/doctos/informes/ensanut_2018_presentacion_resultados.pdf (2020.01.26). 
3. Marchi J, Berg M, Dencker A, et al. Risks associated with obesity in pregnancy, for the mother and baby: a systematic review of reviews. Obes Rev. 2015; 16(8): 621-638, doi: 10.1111/obr.12288, indexed in Pubmed: 26016557.

4. Dabelea D, Snell-Bergeon JK, Hartsfield CL, et al. Kaiser Permanente of Colorado GDM Screening Program. Increasing prevalence of gestational diabetes mellitus (GDM) over time and by birth cohort: Kaiser Permanente of Colorado GDM Screening Program. Diabetes Care. 2005; 28(3): 579-584, doi: 10.2337/diacare.28.3.579, indexed in Pubmed: 15735191.

5. López-de la Peña XA, Cájero Avela JJ, De León Romo LF. Prevalence of gestational diabetes in a group of women receiving treatment at the Mexican Institute of Social Security in Aguascalientes, Mexico. Arch Med Res. 1997; 28: 281-284.

6. Reyes-Muñoz E, Parra A, Castillo-Mora A, et al. Effect of the diagnostic criteria of the International Association of Diabetes and Pregnancy Study Groups on the prevalence of gestational diabetes mellitus in urban Mexican women: a cross-sectional study. Endocr Pract. 2012; 18(2):146-151, doi: 10.4158/EP11167.OR, indexed in Pubmed: 21856596.

7. Kelstrup L, Clausen TD, Mathiesen ER, et al. High prevalence of type 2 diabetes and pre-diabetes in adult offspring of women with gestational diabetes mellitus or type 1 diabetes: the role of intrauterine hyperglycemia. Diabetes Care. 2008; 31(2): 340-346, doi: 10.2337/dc07-1596, indexed in Pubmed: 18000174.

8. Olmos PR, Rigotti A, Busso D, et al. Maternal hypertriglyceridemia: A link between maternal overweight-obesity and macrosomia in gestational diabetes. Obesity (Silver Spring). 2014; 22(10): 2156-2163, doi: 10.1002/oby.20816, indexed in Pubmed: 24957884.

9. Hinkle SN, Rawal S, Liu D, et al. Maternal adipokines longitudinally measured across pregnancy and their associations with neonatal size, length, and adiposity. Int J Obes (Lond). 2019; 43(7): 1422-1434, doi: 10.1038/s41366-018-0255-2, indexed in Pubmed: 30464233.

10. Nanda S, Akolekar R, Sarquis R, et al. Maternal serum adiponectin at 11 to 13 weeks of gestation in the prediction of macrosomia. Prenat Diagn. 2011; 31(5): 479-483, doi: 10.1002/pd.2723, indexed in Pubmed: 21394735.

11. Mazaki-Tovi S, Romero R, Vaisbuch $\mathrm{E}$, et al. Retinol-binding protein 4: a novel adipokine implicated in the genesis of LGA in the absence of gestational diabetes mellitus. J Perinat Med. 2010; 38(2): 147-155, doi: 10.1515/jpm.2010.044, indexed in Pubmed: 20146659.

12. Magee TR, Ross MG, Wedekind L, et al. Gestational diabetes mellitus alters apoptotic and inflammatory gene expression of trophobasts from human term placenta. J Diabetes Complications. 2014; 28(4): 448-459, doi: 10.1016/j.jdiacomp.2014.03.010, indexed in Pubmed: 24768206.

13. Fasshauer $M$, Blüher $M$, Stumvoll $M$. Adipokines in gestational diabetes. Lancet Diabetes Endocrinol. 2014; 2(6):488-499, doi: 10.1016/S22138587(13)70176-1.

14. Ong GKB, Hamilton JK, Sermer M, et al. Maternal serum adiponectin and infant birthweight: the role of adiponectin isoform distribution. Clin Endocrinol (Oxf). 2007; 67(1): 108-114, doi: 10.1111/j.1365-2265 .2007.02846.x, indexed in Pubmed: 17466005.

15. Lowe LP, Metzger BE, Lowe WL, et al. HAPO Study Cooperative Research Group. Inflammatory mediators and glucose in pregnancy: results from a subset of the Hyperglycemia and Adverse Pregnancy Outcome (HAPO) Study. J Clin Endocrinol Metab. 2010; 95(12): 5427-5434, doi: 10.1210/jc.2010-1662, indexed in Pubmed: 20843942.

16. Boeke CE, Mantzoros CS, Hughes MD, et al. Differential associations of leptin with adiposity across early childhood. Obesity (Silver Spring). 2013; 21(7): 1430-1437, doi: 10.1002/oby.20314, indexed in Pubmed: 23408391.

17. Lappas M, Mitton A, Mittion A, et al. In response to oxidative stress, the expression of inflammatory cytokines and antioxidant enzymes are impaired in placenta, but not adipose tissue, of women with gestational diabetes. J Endocrinol. 2010; 204(1): 75-84, doi: 10.1677/JOE-09-0321, indexed in Pubmed: 19833719.

18. Varastehpour A, Radaelli T, Minium J, et al. Activation of phospholipase A2 is associated with generation of placental lipid signals and fetal obesity. J Clin Endocrinol Metab. 2006; 91 (1): 248-255, doi: 10.1210/jc.20050873, indexed in Pubmed: 16249288.
19. Kumarathasan P, Williams G, Bielecki $A$, et al. Characterization of maternal plasma biomarkers associated with delivery of small and large for gestational age infants in the MIREC study cohort. PLoS One. 2018; 13(11): e0204863, doi: 10.1371/journal.pone.0204863, indexed in Pubmed: 30383759.

20. Yeates AJ, McSorley EM, Mulhern MS, et al. Associations between maternal inflammation during pregnancy and infant birth outcomes in the Seychelles Child Development Study. J Reprod Immunol. 2020; 137: 102623, doi: 10.1016/j.jri.2019.102623, indexed in Pubmed: 31710980.

21. Vejrazkova D, Lischkova O, Vankova M, et al. Distinct response of fat and gastrointestinal tissue to glucose in gestational diabetes mellitus and polycystic ovary syndrome. Physiol Res. 2017; 66(2): 283-292, doi: 10.33549/physiolres.933366, indexed in Pubmed: 27982680.

22. Yeung EH, McLain AC, Anderson $N$, et al. Newborn Adipokines and Birth Outcomes. Paediatr Perinat Epidemiol. 2015; 29(4): 317-325, doi: 10.1111/ppe.12203, indexed in Pubmed: 26111443.

23. Wang Yu, Lam KSL, Kraegen EW, et al. Lipocalin-2 is an inflammatory marker closely associated with obesity, insulin resistance, and hyperglycemia in humans. Clin Chem. 2007; 53(1): 34-41, doi: 10.1373/clinchem.2006.075614, indexed in Pubmed: 17040956.

24. Wang Q, Huang R, Yu B, et al. Higher Fetal Insulin Resistance in Chinese Pregnant Women with Gestational Diabetes Mellitus and Correlation with Maternal Insulin Resistance. PLoS ONE. 2013; 8(4): e59845, doi: 10.1371/journal.pone.0059845.

25. Treviño-Garza C, Estrada-Zúñiga $C M$, Mancillas-Adame $L$, et al. Adding Multiple Adipokines into the Model do not Improve Weight Gain Prediction by Leptin Levels in Newborns. J Clin Res Pediatr Endocrinol. 2016; 8(3): 321-324, doi: 10.4274/jcrpe.2693, indexed in Pubmed: 27087431.

26. Fonseca MJ, Santos AC, Fonseca MJ, et al. Umbilical cord blood adipokines and newborn weight change. Arch Gynecol Obstet. 2015; 291(5): 1037-1040, doi: 10.1007/s00404-014-3534-8, indexed in Pubmed: 25384519.

27. Sánchez-Infantes D, Cereijo R, Sebastiani G, et al. Nerve Growth Factor Levels in Term Human Infants: Relationship to Prenatal Growth and Early Postnatal Feeding. Int J Endocrinol. 2018; 2018: 7562702, doi: 10.1155/2018/7562702, indexed in Pubmed: 30675161.

28. Bulló M, Peeraully MR, Trayhurn P, et al. Circulating nerve growth factor levels in relation to obesity and the metabolic syndrome in women. Eur J Endocrinol. 2007; 157(3): 303-310, doi: 10.1530/EJE-06-0716, indexed in Pubmed: 17766712.

29. Flores-Huerta S, Martínez-Salgado H. Peso al nacer de los niños y niñas derechohabientes del Instituto Mexicano del Seguro Social. Bol Med Hosp Infant Mex. 2012; 69: 30-39.

30. Matthews DR, Hosker JP, Rudenski AS, et al. Homeostasis model assessment: insulin resistance and beta-cell function from fasting plasma glucose and insulin concentrations in man. Diabetologia. 1985; 28(7): 412-419, doi: 10.1007/BF00280883, indexed in Pubmed: 3899825.

31. Olmos PR, Borzone GR, Olmos RI, et al. Gestational diabetes and pre-pregnancy overweight: possible factors involved in newborn macrosomia. J Obstet Gynaecol Res. 2012; 38(1): 208-214, doi: 10.1111/j.14 47-0756.2011.01681.x, indexed in Pubmed: 22070342.

32. Lekva T, Norwitz ER, Aukrust $P$, et al. Impact of Systemic Inflammation on the Progression of Gestational Diabetes Mellitus. Curr Diab Rep. 2016; 16(4): 26, doi: 10.1007/s11892-016-0715-9, indexed in Pubmed: 26879309.

33. Alves JG, Souza AS, Figueiroa JN, et al. Visceral Adipose Tissue Depth in Early Pregnancy and Gestational Diabetes Mellitus - a Cohort Study. Sci Rep. 2020; 10(1): 2032, doi: 10.1038/s41598-020-59065-5, indexed in Pubmed: 32029868.

34. Jarvie EM, Stewart FM, Ramsay JE, et al. Maternal Adipose Tissue Expansion, A Missing Link in the Prediction of Birth Weight Centile. J Clin Endocrinol Metab. 2020; 105(3), doi: 10.1210/clinem/dgz248, indexed in Pubmed: 31832635.

35. Perrin EM, O'Shea TM, Skinner AC, et al. Elevations of inflammatory proteins in neonatal blood are associated with obesity and overweight among 2-year-old children born extremely premature. Pediatr Res. 2018; 83(6): 1110-1119, doi: 10.1038/pr.2017.313, indexed in Pubmed: 29244802. 